\title{
Deformations of large fundamental groups
}

\author{
B. de Oliveira * \\ L. Katzarkov ${ }^{\dagger}$ \\ M. Ramachandran
}

\section{Contents}

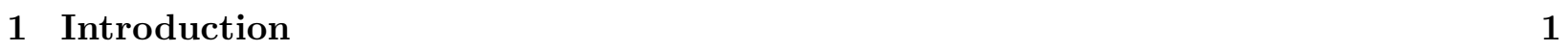

\begin{tabular}{|ll|l}
2 & Tools & 3
\end{tabular}

2.1 The relative Shafarevich morphism in families . . . . . . . . . . . . . . . . . 4

2.2 Volume tools $\ldots \ldots \ldots \ldots \ldots \ldots \ldots \ldots \ldots$

2.3 Topological tools $\ldots \ldots \ldots \ldots \ldots \ldots \ldots \ldots \ldots$

$\begin{array}{lll}3 & \text { Theorems on generically large fundamental groups } & 10\end{array}$

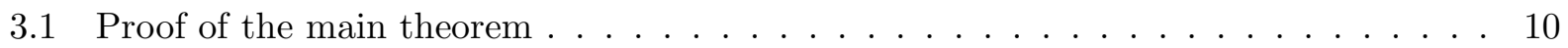

3.2 Deformations of surfaces and $\pi_{1}$-small curves $\ldots \ldots \ldots \ldots \ldots$

\section{Introduction}

The following notion of a variety with a large fundamental group was introduced by Kollár [17] in his study of the Shafarevich uniformization conjecture.

Definition 1 A smooth projective variety $X$ has a large fundamental group if for every irreducible positive dimensional subvariety $Z \subset X$ and every resolution of singularities $\operatorname{res}(Z)$ of $Z$ the image $\operatorname{im}\left[\pi_{1}(\operatorname{res}(Z)) \rightarrow \pi_{1}(X)\right]$ is an infinite group.

For future reference, it will be convenient to give a special name to the subvarieties $Z \subset X$ which obstruct the largeness of the fundamental group.

Definition 2 A subvariety $Z \subset X$ will be called $\pi_{1}$-small if for some (and therefore every) resolution of singularities $\operatorname{res}(Z)$ of $Z$ the image $\operatorname{im}\left[\pi_{1}(\operatorname{res}(Z)) \rightarrow \pi_{1}(X)\right]$ is a finite group.

More generally one says that a projective variety $X$ has a generically large fundamental group if $X$ contains at most countably many $\pi_{1}(X)$-small subvarieties.

\footnotetext{
* Partially supported by NSF Postdoctoral research fellowship

${ }^{\dagger}$ Partially supported by NSF Career Award and A.P.Sloan research fellowship
} 
In this paper we investigate whether the property of a variety having a generically large and large fundamental groups is stable under Kähler deformations.

The main tools we will use are collected in the following three theorems. Their different nature give us extra flexibility to tackle these kind of problems. The statements of the theorems are also of independent interest and have applications outside this article.

Before we state the theorems we will need to introduce some notation. Let $D$ denote a one dimensional complex disk and let $o \in D$ be a marked point. For a smooth proper Kähler morphism $f: X \rightarrow D$ of complex analytic spaces and a point $t \in D$ denote by $X_{t}$ the fiber $f^{-1}(t)$. Given a finitely generated group $\Gamma$ we denote by $\mu: \Gamma \rightarrow \widehat{\Gamma}_{\text {uni }}$ the natural homomorphism from $\Gamma$ to its prounipotent completion. The homomorphism $\mu$ is called the Malcev representation of $\Gamma$. The covering of a space $X$ corresponding to the kernel of the Malcev representation of $X$ is called the Malcev covering of $X$. A group $\Gamma$ is called residually unipotent if the Malcev representation of $\Gamma$ has finite kernel. First we have the following:

Theorem 1 Let $f: X \rightarrow D$ be a Kähler deformation of a Kähler manifold $X_{o}$. Suppose that $\pi_{1}\left(X_{o}\right)$ is residually unipotent, then there exist a complex analytic space $Y$ which fits in a commutative diagram

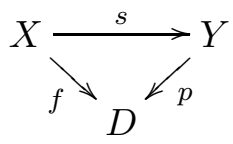

where:

- Both $p$ and $s$ are proper analytic maps with connected fibers.

- The fibers of $p$ are normal projective varieties with large fundamental groups.

- For each $t \in D$ the fibers of $s_{\mid X_{t}}: X_{t} \rightarrow Y_{t}$ are $\pi_{1}\left(X_{t}\right)$-small subvarieties of $X_{t}$.

The following theorem contains our basic volume estimate and gives a restriction on the $\pi_{1}$-small subvarieties that occur in a Kähler deformations of a variety with Stein universal covering.

Theorem 2 Let $f: X \rightarrow D$ be a Kähler deformation of a compact manifold $X_{o}$ and let $\left\{w_{t}\right\}_{t \in D}$ be a family of Kähler metrics along the fibers of $f$. Assume that $X_{o}$ has a Stein universal covering. Fix any $N \in \mathbb{N}$. Then there exists a smaller disk $o \in D(N) \subset D$ such that

$$
\operatorname{vol}_{w_{t}}(Z) \cdot\left(\# \operatorname{im}\left[\pi_{1}(\operatorname{res}(Z)) \rightarrow \pi_{1}\left(X_{t}\right)\right]+1\right)>N
$$

for all $t \in D(N)$ and every $\pi_{1}$-small subvariety $Z \subset X_{t}$.

Finally we have the following statement of a purely topological nature

Theorem 3 Let $f: X \rightarrow T$ be a proper morphism with equidimensional fibers between analytic spaces. Then the subset

$$
\left\{t \in T \mid X_{t} \text { has a } \pi_{1} \text {-small irreducible component }\right\} \subset T
$$

is a closed analytic subset in $T$. 
The main objective of the present paper is to use the results stated above to study the behavior of large fundamental groups of Kähler surfaces under deformations. In this direction we obtain the following:

Theorem 4 Let $X$ be a Kähler surface and assume that the Malcev covering of $X$ is an infinite covering. Then the property of $X$ having a generically large fundamental group is stable under small Kähler deformations.

As a consequence we have the following:

Corollary 5 Let $X$ be a compact Kähler surface, let $U$ be a finite dimensional linear unipotent group, and let $\rho: \pi_{1}(X) \rightarrow U$ be a representation with an infinite image. Suppose that the covering of $X$ defined by $\operatorname{ker}(\rho)$ is Stein. Then any small Kähler deformation of $X$ has a Stein universal covering.

We make the following conjectures:

Conjecture 1.1 Let $X$ be a Kähler manifold and let $\rho: \pi_{1}(X) \rightarrow G L(n, \mathbb{C})$ be an infinite linear representation. Then having a generically large fundamental group is stable under small Kähler deformations of $X$.

Conjecture 1.2 Let $X$ be a compact Kähler manifold whose universal covering is Stein. Then small Kähler deformations of $X$ have large fundamental group.

If the Shafarevich conjecture is correct this implies

Conjecture 1.3 If $X$ is a compact Kähler manifold with a Stein universal cover, then every small deformation of $X$ also has a Stein universal cover.

It was shown in [4] that Conjecture 1.3 is not correct without the Kähler hypothesis. We also note that what was lost in that example was not the property of the fundamental group being large but the holomorphic convexity.

The paper is organized as follows. In section two we describe the methods and the main technical tools used in the proofs of the above theorems. In section three we give the proofs of Theorems 2 and 1 and some corollaries.

Acknowledgments: We thank F.Bogomolov, M.Gromov, J.Kollár, T.Pantev and C.Simpson for useful conversations.

\section{Tools}

In this section we describe the basic techniques used in the proof of the main theorem. We start with some facts from nonabelian Hodge theory. 


\subsection{The relative Shafarevich morphism in families}

Since the $\pi_{1}$-small subvarieties are the obstruction to largeness of the fundamental group, we have to learn how to contract such subvarieties in the absolute and relative context. In the absolute case the answer is given by the Shafarevich holomorphic convexity conjecture which can be stated as follows:

Conjecture 2.1 Let $X$ be a smooth complex projective variety. Then:

(1) There exists a normal analytic space $\boldsymbol{S h}(\widetilde{X})$ with no compact complex subspaces and a proper morphism with connected fibers sh $: \widetilde{X} \rightarrow \boldsymbol{S h}(\widetilde{X})$. This morphism is known as the CartanRemmert reduction of $\widetilde{X}$.

(2) $\boldsymbol{S h}(\widetilde{X})$ is a Stein space.

The validity of the Shafarevich conjecture has been recently established in several classes of examples due to the efforts of F.Bogomolov, F.Campana, P.Eyssidieux, L.Katzarkov, J.Kollár, T.Napier, R.Narasimhan, M.Nori, T.Pantev, M.Ramachandran (see e.g. [8], [9], [15], [14], [17], [16], [21]). The strongest result to date is due to Eyssidieux [8], who showed that the Shafarevich conjecture is true for the covering corresponding to the intersections of the kernels of all reductive representations of $\pi_{1}(X)$ in some $G L(n, \mathbb{C})$.

A starting point of most of the recent research on the Shafarevich conjecture is the important remark of Kollár [16] and [17] who observed that condition (1) above is equivalent to:

$\left(1^{\prime}\right)$ There exists a normal variety $\boldsymbol{S h}(X)$ and a proper morphism with connected fibers $\boldsymbol{s h}: X \rightarrow$ $\boldsymbol{S h}(X)$, which contracts exactly $\pi_{1}(X)$-small subvarieties of $X$.

Clearly if such a $\boldsymbol{S h}(X)$ exists, it will have a large fundamental group. Therefore property $\left(1^{\prime}\right)$ will allow one to reduce any geometric question related to the fundamental group to a question about a variety with a large fundamental group. Much work has been done on finding various sufficient conditions for the validity of $\left(1^{\prime}\right)$. The only result in that direction which works in full generality is due to Campana [6] and Kollár [17], [16] who have shown that one can always find a proper rational map sh : $X \rightarrow \operatorname{Sh}(X)$ with connected fibers which contracts only $\pi_{1}(X)$-small subvarieties and such that $\operatorname{Sh}(X)$ has a generically large fundamental group.

In what follows we will need Kollár's notion of $H$-Shafarevich morphism which is a slight generalization of $\left(1^{\prime}\right)$ and which we recall next. We will say that a subgroup $\Gamma \subset \pi_{1}(X)$ is almost contained in $H$ if the intersection $\Gamma \cap H$ has finite index in $\Gamma$ and we will write $\Gamma \lesssim H$. The $H$-Shafarevich morphism is a proper map with connected fibers $\boldsymbol{s} \boldsymbol{h}^{H}: X \rightarrow \boldsymbol{S} \boldsymbol{h}^{H}(X)$ to a normal variety $\boldsymbol{S} \boldsymbol{h}^{H}(X)$ which contracts exactly the subvarieties $Z \subset X$ having the property that $\operatorname{im}\left[\pi_{1}(\operatorname{res}(Z)) \longrightarrow \pi_{1}(X)\right] \lesssim H$.

Recently using ideas and techniques from non-abelian Hodge theory developed by Corlette [7], Gromov and Schoen [10] and Simpson [24] the following statements were proved (see [12], [14]).

Theorem 2.2 ([12, 14]) Let $X$ be a projective manifold and let $\rho: \pi_{1}(X) \rightarrow G L(n, \mathbb{C})$ be a linear representation with kernel $H$. Then there exists a blow-up $\widehat{X}$ of an étale finite cover of $X$ and a projective morphism $\operatorname{alb}_{\rho}: \widehat{X} \rightarrow \operatorname{Alb}_{\rho}(X)$ satisfying: 
(i) $\operatorname{Alb}_{\rho}(X)$ is a smooth fibration of abelian varieties over a variety of general type.

(ii) alb $_{\rho}$ contracts exactly the subvarieties $Z \subset \widehat{X}$ having the property that

$$
\operatorname{im}\left[\pi_{1}(\operatorname{res}(Z)) \longrightarrow \pi_{1}(X)\right] \lesssim H .
$$

In particular the $H$-Shafarevich morphism $\boldsymbol{s h}^{H}: \widehat{X} \rightarrow \boldsymbol{S} \boldsymbol{h}^{H}(\widehat{X})$ exists and coincides with the Stein factorization of $\operatorname{alb}_{\rho}$.

As an immediate consequence of this theorem one shows

Corollary 2.3 (匹12, 14]) Let $X$ be a Kähler manifold and let $\rho: \pi_{1}(X) \rightarrow G L(n, \mathbb{C})$ be an almost faithful representation. Then the Shafarevich morphism sh: $X \rightarrow \boldsymbol{S h}(X)$ exists and $\boldsymbol{S h}(X)$ and $\operatorname{Sh}(X)$ are birationally isomorphic. Furthermore if $X$ is a surface, then the Shafarevich conjecture is true for $X$.

In order to understand how the varieties with large fundamental groups vary in families one has to understand how the Shafarevich variety $\boldsymbol{S h}(X)$ of a projective manifold $X$ changes under small deformations of the complex structure of $X$. We believe that the following Conjecture should be true:

Conjecture 2.4 Let $f: X \rightarrow D$ be a Kähler deformation of a Kähler manifold $X_{o}$ over D. Let $H \subset \pi_{1}(X)=\pi_{1}\left(X_{o}\right)$ be a subgroup for which the $H$-Shafarevich morphism $X_{o} \rightarrow \boldsymbol{S h}^{H}\left(X_{o}\right)$ exists. Then there exist

a) a finite base change $\Delta \rightarrow D$ branched at $o \in D$,

b) a relative Shafarevich variety $p_{H}: S \boldsymbol{h}^{H}\left(X \times_{D} \Delta\right) \rightarrow \Delta$ and a morphism

$$
\operatorname{sh}^{H}: X \times_{D} \Delta \rightarrow \operatorname{Sh}^{H}\left(X \times_{D} \Delta\right),
$$

so that for all $t \in \Delta$ the restriction $\boldsymbol{s h}_{\mid X_{t}}^{H}: X_{t} \rightarrow \boldsymbol{S h}^{H}\left(X_{t}\right)$ is the H-Shafarevich morphism for $X_{t}$.

Unfortunately, the methods employed in [12, 14] for proving Theorem 2.2 and Corollary 2.3 do not extend to the relative situation. The main problem which prevents one from proving Conjecture 2.4 by the methods of [12, 14] is analytic in nature. More specifically, recall that for a projective manifold $X$ the Shafarevich variety $\operatorname{Sh}^{H}(X)$ corresponding to the kernel $H=\operatorname{ker}(\rho)$ of a reductive representation $\rho: \pi_{1}(X) \rightarrow G L(n, \mathbb{C})$ is built as a Castelnuovo-de Franchis reduction of $X$ with respect to a certain system of multivalued holomorphic one forms on $X$ These forms are cnstructed as pullbacks of some constant one forms on a non-positively curved space by a $\rho$ equivariant pluriharmonic map. In the situation when $f: X \rightarrow D$ is a smooth projective morphism it is very hard to ensure the global existence of such $\rho$-equivariant harmonic map and even when exists it is hard to show that this map has reasonable regularity properties. Some partial results in that direction have been obtained in [20] but they require that some very stringent conditions are imposed on the boundary $\partial X$ of $X$ and do not seem to be immediately applicable to the situation at hand. In particular, even when one can prove that the multivalued forms giving rise to $\operatorname{Sh}^{H}\left(X_{t}\right)$ exist for every $X_{t}$, it seems quite hard to check that these forms will vary holomorphically with $t \in D$. 
On the other hand, there are several instances in which one can prove Conjecture 2.4 under some mild additional assumption on the group $H$ or on the Kähler deformation $f: X \rightarrow D$. For example Theorem 1 asserts validity of Conjecture 2.4 under the additional condition that $H$ is the kernel of the Malcev representation of $\pi_{1}(X)$. Slightly more generally we have the following

Claim 2.5 Let $f: X \rightarrow D$ be a morphism with smooth projective connected fibers. Let $U$ be any proalgebraic group which is a quotient of the prounipotent completion $\hat{\pi}_{\text {uni }}(X)$ of the fundamental group of $X$. Let $H=\operatorname{ker}\left[\pi_{1}(X) \rightarrow U\right]$. Then the Shafarevich morphism sh $^{H}: X_{o} \rightarrow \boldsymbol{S h}^{H}\left(X_{o}\right)$ exists and Conjecture 2.4 holds for $f: X \rightarrow D$ and $H$.

Proof. Let $\Gamma:=\operatorname{im}\left[\pi_{1}(X) \rightarrow U\right]$ and consider the image $L:=\operatorname{im}\left[h_{1}\left(X_{o}, \mathbb{Z}\right) \rightarrow \Gamma /[\Gamma, \Gamma]\right]$. The finitely generated abelian group $L$ corresponds to some abelian variety $A_{o}$ which is a quotient $\operatorname{Alb}\left(X_{o}\right) \rightarrow A_{o}$ of the Albanese variety of $X_{o}$. As explained in [13, Section 2] the existence of a MHS on $\hat{\pi}_{\text {uni }}(X)$ and the strictness property of morphism of MHS implies that the Stein factorization of the natural morphism $X_{o} \rightarrow \operatorname{Alb}\left(X_{o}\right) \rightarrow A_{o}$ is precisely the $H$-Shafarevich morphism

$$
\boldsymbol{s h ^ { H }}: X_{o} \rightarrow \boldsymbol{S h}^{H}\left(X_{o}\right) .
$$

On the other hand, since $f: X \rightarrow D$ is smooth, projective and with connected fibers we may assume that (after maybe shrinking $D) f$ admits an analytic section and that we have a relative Albanese variety $\operatorname{Alb}(X / D) \rightarrow D$ and a relative Albanese morphism

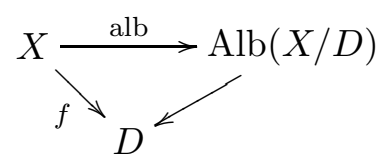

Let $A \rightarrow D$ be the family of abelian varieties which is the quotient of $\operatorname{Alb}(X / D)$ corresponding to $L$. Again by [13, Section 2] the Stein factorization of the composition $X \rightarrow \operatorname{Alb}(X / D) \rightarrow A$ restricts to the $H$-Shafarevich morphism on each $X_{t}$. The claim is proven.

Another special case of Conjecture 2.4 can be obtained as follows:

Claim 2.6 Let $f: X \rightarrow D$ is a smooth projective morphism with connected fibers and suppose that there exist a morphism with connected fibers of smooth projective varieties $\pi: M \rightarrow S$ and a fiber product diagram

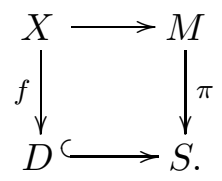

Assume further that $H$ is the kernel of the pullback of some finite dimensional linear representation $\rho: \pi_{1}(M) \rightarrow G L(n, \mathbb{C})$ of $\pi_{1}(M)$ via the natural map $\pi_{1}(X) \rightarrow \pi_{1}(M)$. Then there exists a blow-up $\widehat{X}$ of a finite étale covering of $X$, so that $H$-Shafarevich morphism exists for $\widehat{X}_{o}$ and Conjecture 2.4 holds for $\hat{f}: \widehat{X} \rightarrow D$ and $H$. 
Proof. For any blow-up $\widehat{X}$ of a finite étale covering of $X$, Theorem 2.2 implies that the $H$ Shafarevich morphism for $\widehat{X}_{o}$ exist. To check the validity of Conjecture 2.4 note that by 24, Theorem 3] we can deform $\rho$ to a representation $\lambda$ underlying a complex variation of Hodge structures and so the restriction of $\lambda$ to each $\pi_{1}\left(X_{t}\right)$ will be a representation underlying a complex variation of Hodge structures on $X_{t}$. Now consider the Stein factorization $s: X \rightarrow Y$ of the holomorphic horizontal map corresponding to the periods of these variations of Hodge structures. As argued in [22], the maps $s_{\mid X_{t}}: X_{t} \rightarrow Y_{t}$ will be precisely the $\operatorname{ker}\left(\lambda_{\mid \pi_{1}(X)}\right)$-Shafarevich morphisms on each $X_{t}$. By replacing $X$ with a blow-up $\widehat{X}$ of a finite étale covering of $X$ we may assume that $Y$ is smooth. Finally, as explained in the proof of [12, Theorem 4.4], one has a dihotomy - either $s_{\mid \widehat{X}_{t}}$ is in fact the $H$-Shafarevich on $\widehat{X}_{t}$ or $\lambda$ is defined over a number field and some finite direct sum of conjugates of $\lambda$ underlies a polarized integral variation of Hodge structures $\mathbb{V}$ of weight one on $Y$. Furthermore the strictness property of maps of MHS implies [12, Theorem 4.4] that the relative Albanese morphism $a: X \rightarrow A$ for the family of abelian varieties $A$ on $Y$ corresponding to $\mathbb{V}$ contracts precisely the subvarieties $Z \subset X$ for which $\operatorname{im}\left[\pi_{1}\left(\operatorname{res}(Z) \rightarrow \pi_{1}(X)\right] \lesssim \operatorname{ker}\left(\rho_{\mid \pi_{1}(X)}\right)\right.$. Thus $\boldsymbol{s} \boldsymbol{h}^{H}: X \rightarrow \boldsymbol{S} \boldsymbol{h}^{H}(X)$ exists and coincides with the Stein factorization of $a$. The claim is proven.

Remark 2.7 It is worth observing that the relative version of Kollár's Shafarevich map does exist and is relatively easy to construct. In other words, for any analytic map $f: X \rightarrow D$ whose fibers are connected Kähler manifolds and any subgroup $H \subset \pi_{1}(X)$ we can find a complex analytic space $\operatorname{Sh}(X)$ which fits in a commutative diagram

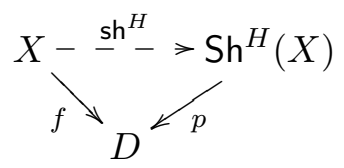

so that $\operatorname{sh}_{\mid X_{t}}^{H}: X_{t} \rightarrow \mathrm{Sh}^{H}(X)_{t}$ is a Kollár's Shafarevich map for all $t \in D$. Indeed, in order to construct $\operatorname{Sh}(X)$ we only have to repeat the argument in the proof of [17, Theorem 3.6]. The only ingredient in this proof which does not automatically carry over to the analytic situation is the existence of a locally topologically trivial family of normal cycles on $X$. This however is guaranteed in the relative situation (possibly after a finite base change) by [23].

Note also that since by construction $\operatorname{sh}^{H}$ fails to contract at most countably many compact subvarieties $Z \subset X$ for which $\operatorname{im}\left[\pi_{1}(\operatorname{res}(Z)) \rightarrow \pi_{1}(X)\right] \lesssim H$, it follows that for all but countably many $t \in D$ the rational map $\operatorname{sh}_{\mid X_{t}}^{H}: X_{t} \rightarrow \operatorname{Sh}^{H}(X)_{t}$ will actually coincides with the Shafarevich morphism for $X_{t}$. If in addition $H$ is the kernel of a finite dimensional linear representation of $\pi_{1}(X)$ we can apply Theorem 2.2 to conclude that the $H$-Shafarevich morphism exists fiber by fiber and so in each of the countably many fibers $\operatorname{Sh}^{H}(X)_{t}$ of $\operatorname{Sh}^{H}(X)$ which do not equal $\boldsymbol{S} \boldsymbol{h}^{H}\left(X_{t}\right)$ we will have at most finitely many subvarieties $Z \subset \boldsymbol{S} \boldsymbol{h}^{H}\left(X_{t}\right)$ for which $\operatorname{im}\left[\pi_{1}(\operatorname{res}(Z)) \rightarrow \pi_{1}\left(\boldsymbol{S h}^{H}\left(X_{t}\right)\right)\right] \lesssim H$.

\subsection{Volume tools}

In this section we study the Kähler deformations of compact Kähler manifolds $X_{O}$ whose universal coverings are Stein. A Kähler deformation of $X_{o}$ means a Kähler manifold $X$ and a smooth proper 
morphism $f: X \rightarrow D$ to the one dimensional complex disk $D$ with $X_{o}=f^{-1}(o)$, s.t. the induced Kähler metric on the fibers $X_{t}$ varies smoothly.

¿From now on $\widetilde{X}$ will denote the universal covering of $X$ and $\sigma: \widetilde{X} \rightarrow X$ denotes the respective covering map. If $\widetilde{Z}$ is a compact subvariety of $\widetilde{X}$ we denote by $Z$ its reduced image by $\sigma$. We denote the degree of the mapping $\sigma_{\mid \widetilde{Z}}: \widetilde{Z} \rightarrow Z$ by $n_{\widetilde{Z}}$. Clearly the fact that $\widetilde{Z}$ is compact is equivalent to $\operatorname{im}\left[\pi_{1}(\operatorname{res}(Z)) \rightarrow \pi_{1}(X)\right]$ being finite. We denote the order of this subgroup of $\pi_{1}(X)$ by $\# \operatorname{im}\left[\pi_{1}(\operatorname{res}(Z)) \rightarrow \pi_{1}(X)\right]$. We introduce a notation:

Definition 3 Let $X$ be a complex manifold and let $i: Z \hookrightarrow X$ be a compact subvariety of dimension d. If $w$ is a Kähler form on $X$ define

$$
\operatorname{vol}_{w}(Z)=\frac{1}{d !} \int_{Z-\operatorname{Sing} Z}\left(i^{*} w\right)^{d}=\left\langle[w]^{d},[Z]\right\rangle,
$$

$[w] \in H^{2}(X, \mathbb{C})$ is the Kähler class and $[Z] \in H_{2 d}(X, \mathbb{Z})$ is the homology class of $Z$. Similarly if $L$ is a positive line bundle on $X$ define

$$
\operatorname{vol}_{L}(Z)=\int_{Z-\operatorname{Sing} Z} d V_{L}=\frac{1}{d !} \int_{Z-\operatorname{Sing} Z}\left(i^{*} \nu\right)^{d}=\operatorname{deg}_{Z}(L)
$$

where $d V_{L}$ is the volume element coming from a metric associated to a curvature form $\nu$ for $L$.

Let $f: X \rightarrow D$ be a deformation of the compact manifold $X_{o}$ over the disc $D$. Then we also have a deformation $\tilde{f}: \widetilde{X} \rightarrow D$ of the universal covering $\widetilde{X}_{o}$ of $X_{o}$. This deformation is naturally $C^{\infty}$ trivial. Pick a general point $x(o) \in \widetilde{X}_{o}$ and let $\Sigma_{x(o)} \subset \widetilde{X}$ be a fundamental domain for the $\pi_{1}\left(X_{o}\right)$ action on $X_{o}$ containing $x(o)$. Using the $C^{\infty}$ trivialization $\tilde{X} \cong X_{o} \times D$ of $\tilde{f}: \widetilde{X} \rightarrow D$ we can extend $x(o)$ to a smooth section $x: D \rightarrow \widetilde{X}$ of the structure map $\tilde{f}$. Also since $\pi_{1}(X)=\pi_{1}\left(X_{o}\right)$ the subset $\Sigma:=\Sigma_{x(o)} \times D \subset \widetilde{X}$ will be a fundamental domain for the $\pi_{1}(X)$ action on $\tilde{X}$ containing $x(o)$. Therefore the subset $\Sigma_{x(t)}=\Sigma \cap X_{t}$ will be a fundamental domain for the $\pi_{1}\left(X_{t}\right)$ action on $\widetilde{X}_{t}$ containing $x(t)$. with this notation established we are now ready to prove:

Claim 2.8 Let $f: X \rightarrow D$ be a Kähler deformation of $X_{o}$ and let $\left\{w_{t}\right\}_{t \in D}$ be a smooth family of Kähler metrics along the fibers of $f$. Assume that $X_{o}$ has a Stein universal covering. Fix any $N \in \mathbb{N}$. Then there exists a smaller disk $D(N) \subset D$ centered at o so that

$$
\operatorname{vol}_{\tilde{w}_{t}}(\widetilde{Z})>N
$$

for all compact subvarieties $\widetilde{Z} \subset \widetilde{X}_{t}$ where $\widetilde{X}_{t}$ is the universal covering of $X_{t}$ and $\tilde{w}_{t}$ is the pullback of $w_{t}$.

Proof. The deformation $\tilde{f}: \widetilde{X} \rightarrow D$ of the Stein manifold $X_{o}$ is locally pseudo-trivial [1], i.e. for any relatively compact open subset $U_{o} \subset \tilde{X}_{o}$ we can find an open set $U \subset X$ such that $U \cap \widetilde{X}_{o}=U_{o}$, and for which the family $f_{\mid U}: U \rightarrow D$ defines a holomorphically trivial deformation of $U_{o}$. The open sets $U_{t}=U \cap \widetilde{X}_{t}$ are obtained by flowing $U_{o}$ along a holomorphic vector field. Choose $U_{o}$ so that it contains a very big ball $B_{x(o)}(r), r>>0$, around a point $x(o) \in \widetilde{X}_{o}$. Since a fundamental domain $\Sigma_{x(o)}$ containing $x(o)$ is relatively compact we have $\Sigma_{x(o)} \subset B_{x(o)}\left(r^{\prime}\right)$, for some $r^{\prime}$. Since $U_{t}, x(t), \Sigma_{x(t)}$ and $w_{t}$ vary smoothly with $t$ we can shrink $D$ so that for all $t \in D$ we have 
1) $B_{x(t)}(r) \subset U_{t}$, and

2) $\Sigma_{x(t)} \subset B_{x(t)}\left(r^{\prime}\right)$.

Condition 1) implies that all compact subvarieties $\widetilde{Z}$ of $\widetilde{X}_{t}$ which intersect the fundamental domain $\Sigma_{x(t)}$ can not be contained in $B_{x(t)}(r)$. This follows from $U_{t}$ being biholomorphic to an open subset of the Stein space $\widetilde{X}_{o}$. Condition 2 ) states that any subvariety $\widetilde{Z} \subset X_{t}$ which intersects $\Sigma_{x(t)}$ must have $\left[r-r^{\prime}\right]$ (the integer part of $r-r^{\prime}$ ) disjoint pieces of the type $\widetilde{Z} \cap B_{x(t)}(1)$.

Recall the standard fact saying that for any Kähler manifold $X$ of bounded geometry, any $k$ dimensional subvariety $S \subset X$ and any points $x \in X$ one has $\operatorname{vol}_{2 k}\left(S \cap B_{x}(1)\right)>C>0$, where $C$ depends only on the bound for the geometry of $X$. Since every subvariety $\widetilde{Z} \subset X_{t}$ can be translated to asubvariety intersecting $\Sigma_{x(t)}$ the claim follows from:

$$
\operatorname{vol}_{\tilde{w}_{t}}(\widetilde{Z})>\sum_{i=1}^{\left[r-r^{\prime}\right]} \operatorname{vol}_{\tilde{w}_{t}}\left(\widetilde{Z} \cap B_{x(t)}(1)\right)>\left[r-r^{\prime}\right] . C
$$

and the fact that $r$ can be made as big as wanted by making $D$ sufficiently small. Notice that the minimum $C=\inf _{t \in D}\left\{C_{t}\right\}$ of the geometry bounds $C_{t}$ described above is not zero since the metrics vary smoothly.

\subsection{Topological tools}

In this subsection we prove Theorem 3. In the proof we use the topological stratification and the "lower semicontinuity" property of the homotopy groups of the fibers of a proper morphism.

Proof of Theorem 3. Let $f: X \rightarrow T$ be a proper morphism with equidimensional fibers between analytic spaces. Let $X_{t}=\cup_{l=1}^{k_{t}} X_{t}^{l}$ be the irreducible decomposition of the fiber $X_{t}$. Let $o \in T$ be a point such that the fiber $X_{o}$ does not have any $\pi_{1}$-small irreducible component. We have

$$
\# \operatorname{im}\left[\pi_{1}\left(\operatorname{res}\left(X_{t}^{l}\right) \rightarrow \pi_{1}(X)\right]=\infty .\right.
$$

for all $t \in U$ and all $1 \leq l \leq k_{t}$. Due to [25, Theorems $\left.2.2 ; 3.3 ; 4.14\right]$ and the fact that $f$ is proper we can find an open neighborhood $U$ of $o \in T$ and a finite stratification that is $U=\cup_{i} S_{i}$ of $U$ by locally closed irreducible subsets so that $f_{i}:=f_{\mid f^{-1}\left(S_{i}\right)}: f^{-1}\left(S_{i}\right) \rightarrow S_{i}$ are topologically trivial fibrations. Now pick a neighborhood $o \in V \subset U$ s.t. $S_{i} \cap V \neq \varnothing$ iff $o \in \bar{S}_{i}$. Consider the morphisms $\bar{f}_{i}:=f_{\mid f^{-1}\left(\bar{S}_{i}\right)}: f^{-1}\left(\bar{S}_{i}\right) \rightarrow \bar{S}_{i}$. The theorem will follow if we show that the general fiber component of each of the morphisms $\bar{f}_{i}$ is not $\pi_{1}$-small.

Pick an irreducible component of $f^{-1}\left(\bar{S}_{i}\right)$ that dominates $\bar{S}_{i}$. Let $\left.\widehat{f^{-1}\left(\bar{S}_{i}\right.}\right)$ and $\widehat{\bar{S}}_{i}$ denote the normalizations of $f^{-1}\left(\bar{S}_{i}\right)$ and $\bar{S}_{i}$ respectively. We get an induced morphism of normal irreducible varieties $\hat{\bar{f}}_{i}:{\widehat{f}-1\left(\bar{S}_{i}\right)} \rightarrow \widehat{\bar{S}}_{i}$. Notice that the irreducible components of the fiber $\hat{\bar{f}}_{i}^{-1}(t)$ are partial normalizations of some irreducible components of the fiber $X_{t}=f^{-1}(t)$. Hence the hypothesis of the theorem is still valid for the morphism $\hat{\bar{f}}_{i}$. By construction of the stratification we know that if $S_{j} \neq S_{i}$ are two strata such that $S_{j} \subset \bar{S}_{i}$ is of minimal codimension then the fiber of $\hat{\bar{f}}_{i}$ over a point in $S_{j}$ will be a deformation retract of the fiber of $\hat{\bar{f}}_{i}$ over a point in $S_{i}$. In particular if $Y$ is the normalization of a component of $\hat{\bar{f}}_{i}^{-1}(t), t \in S_{i}$, then $\operatorname{im}\left[\pi_{1}(Y) \rightarrow \pi_{1}(X)\right]$ coincides with 
$\operatorname{im}\left[\pi_{1}(Z) \rightarrow \pi_{1}(X)\right]$, where $Z$ is the normalization of some component of $\hat{\bar{f}}_{i}^{-1}(s)$ for some point $s \in S_{j}$. Due to this we may assume that $o \in S_{i}$ and so it suffices to check the validity of the theorem for a $f: X \rightarrow T$ satisfying: (a) $f$ is a proper morphism of irreducible normal complex spaces which; (b) $f$ a topological fiber bundle over a dense open subset $\dot{T}$ of $T$; (c) $o \in \dot{T}$. But in this situation the statement of the theorem is obvious since $f$ is topologically locally trivial around the point $o$.

For future reference we also give a proof of the following topological result which is a straightforward generalization of the results of [19], [5], and 17].

Proposition 2.9 Let $X$ be a normal projective variety. Then $\pi_{1}(X)$ is finite if and only if $X$ is chain connected via $\pi_{1}$-small curves.

Proof. One implication is obvious. Suppose that $X$ is chain connected via $\pi_{1}$-small curves. By [17, Theorem 1.8] there exists an open subset $\dot{X}$ and a proper morphism $f: \dot{X} \rightarrow Z$, the Shafarevich map, such that if $z \in Z$ then $X_{z}=f^{-1}(z)$ is $\pi_{1}$-small. This map has also the property that if $z \in Z$ is very general all normal cycles which are $\pi_{1}$-small and intersect $X_{z}$ must be contained in $X_{z}$. Pick a point $x \in X_{z}$, with $z$ very general. Let $y$ be any other point in $X$. There is a chain of $\pi_{1}$-small curves $\left\{C_{1}, C_{2}, \ldots, C_{k}\right\}$ with $C_{i} \cap C_{i+1} \neq \varnothing, x \in C_{1}$ and $y \in C_{k}$. The property of the Shafarevich map mentioned above implies that $C_{1} \subset X_{z}$, which in turn implies $C_{2} \subset X_{z}$ and so on and hence $y \in X_{z}$. This implies $f(X)=$ point and we are done.

\section{Theorems on generically large fundamental groups}

\subsection{Proof of the main theorem}

In this subsection we prove Theorem 2 and Theorem 6

Proof of Theorem 2. Let $X_{o}$ be a compact Kähler manifold with a Stein universal cover and let $f: X \rightarrow D$ be a Kähler deformation of $X_{o}$. Let as before $\left\{w_{t}\right\}_{t \in D}$ denote a smooth family of Kähler metrics along the fibers of $f$. Fix $N \in \mathbb{N}$. According to Claim 2.8 we can find a smaller disk $o \in D(N) \subset D$ so that for any $t \in D(N)$ and all compact subvarieties $\widetilde{Z} \subset \widetilde{X}_{t}$ we have $\operatorname{vol}_{\tilde{w}_{t}}(\widetilde{Z})>N$. The theorem now follows from the obvious identities

$$
\operatorname{vol}_{\tilde{w}_{t}}(\widetilde{Z})=n_{\widetilde{Z}} \cdot \operatorname{vol}_{w_{t}}(Z)
$$

and

$$
n_{\widetilde{Z}}=\# \operatorname{im}\left[\pi_{1}(\operatorname{res}(Z)) \rightarrow \pi_{1}\left(X_{t}\right)\right]
$$

We also have some immediate corollaries:

Corollary 3.1 Let $X_{o}$ be a compact Kähler manifold with a Stein universal cover. Suppose $f$ : $X \rightarrow D$ is a deformation of $X_{o}$ and let $L \rightarrow X$ be a $f$-ample line bundle. Then for any $N \in \mathbb{N}$ 
there exists a smaller disk $o \in D(N) \subset D$ so that for any $t \in D(N)$ and all compact subvarieties $\widetilde{Z} \subset \widetilde{X}_{t}$ we have

$$
\operatorname{deg}_{L_{t}}(Z) \cdot\left(\# \operatorname{im}\left[\pi_{1}(\operatorname{res}(Z)) \rightarrow \pi_{1}\left(X_{t}\right)\right]+1\right)>N
$$

where $L_{t}=L_{\mid X_{t}}$.

Proof. It is straightforward consequence of Theorem 2 applied to the family of Kähler metrics corresponding to the curvature form of relative ample line bundle $L$.

Corollary 3.2 Let $X_{o}$ be a compact Kähler manifold with a Stein universal cover and let $f: X \rightarrow$ $D$ be a Kähler deformation of $X_{o}$. Suppose that the order of all finite subgroups in $\pi_{1}\left(X_{o}\right)$ is bounded from above. Then for any $N \in \mathbb{N}$ we can find a smaller disk $o \in D(N) \subset D$ so that for any $t \in D(N)$ and all $\pi_{1}$-small subvarieties $Z \subset X_{t}$ we have $\operatorname{vol}_{w_{t}}(Z)>N$.

Proof. Let $b$ be the upper bound for the order of the finite subgroups of $\pi_{1}\left(X_{o}\right)$. By Theorem 2 we can find a disk $o \in D^{\prime} \subset D$ so that for any $t \in D^{\prime}$ and any $\pi_{1}$-small subvariety $Z \subset X_{t}$ we have

$$
\operatorname{vol}_{w_{t}}(Z) \cdot\left(\# \operatorname{im}\left[\pi_{1}(\operatorname{res}(Z)) \rightarrow \pi_{1}(X)\right]+1\right)>N \cdot b .
$$

But by assumption \# $\operatorname{im}\left[\pi_{1}(\operatorname{res}(Z)) \rightarrow \pi_{1}(X)\right]<b$ and so the corollary is proven.

The above corollary explains that for a small Kähler (projective) deformations of a Kähler (projective) manifold as in the corollary the property of having a large fundamental group can be only broken by subvarieties with very big volume (degree). Hence if we have an upper bound for the degree of the subvarieties that can violate the property of a large fundamental group, we can show that large fundamental groups are preserved under small Kähler deformations. We use this idea to give a proof of Theorem 1 . In fact we can proof the following general

Proposition 3.3 Let $f: X \rightarrow D$ be a Kähler deformation of a Kähler surface $X$ and assume that $H \subset \pi_{1}(X)$ is a subgroup of infinite index such that the $H$-Shafarevich morphism for $X_{o}$ exists and Conjecture 2.4 holds for $f: X \rightarrow D$ and $H$. Assume also that $X_{O}$ has a generically large fundamental group. Then $X_{t}$ has a generically large fundamental group for all $t$ in a small analytic neighborhood of $o \in D$.

Remark 3.4 Note that the previous proposition in combination with Claim 2.5 implies Theorem 4. Moreover Claims 2.5 and 2.6 give other situations to which we can apply Proposition 3.3 to conclude that the property of having a generically large fundamental group will be preserved under deformations

We will give two different proofs Proposition 3.3. (All surfaces are assumed to be compact and connected unless stated otherwise.)

First Proof. The existence of a relative Shafarevich morphism plus the "lower semicontinuity" of the the homotopy groups in a family gives the first proof.

Let $X_{o}$ be a compact Kähler surface and let $\rho: \pi_{1}\left(X_{o}\right) \rightarrow G L(n, \mathbb{C})$ be an infinite linear representation with kernel $H$. Let $f: X \rightarrow D$ be a deformation of $X_{o}$. By Theorem 1 there exists 
a relative $H$-Shafarevich morphism for the family $f: X \rightarrow D$ possibly after a finite base change. Since the stability of $\pi_{1}$-small curves cannot be affected by a base change we may assume without a loss of generality that we have a relative $H$-Shafarevich morphism $s \boldsymbol{h}^{H}: X \rightarrow \boldsymbol{s h}^{H}(X)$ for $X$ itself. Let $g$ denote the product morphism $g:=\boldsymbol{s h}^{H} \times f: X \rightarrow \boldsymbol{S h}^{H}(X) \times D$.

Since the $\pi_{1}$-small curves must lie in the fibers of $g$, we need to show that there exists an open neighborhood $U, o \in U \subset D$ s.t. for all $t \in U$ the fibers $X_{y, t}=g^{-1}(y, t)$ satisfy:

$$
\operatorname{im}\left[\pi_{1}\left(\operatorname{res}\left(X_{(y, t)}\right)\right) \rightarrow \pi_{1}(X)\right]=\infty .
$$

There are two cases:

Case 1. All fibers $X_{(y, o)}, y \in \boldsymbol{S h}^{H}(X)$ are zero dimensional. The dimension of the fibers of $f$ is an upper-semicontinuous function in the analytic topology of $g(X)$. This implies that there exists an open neighborhood $U$ of $o \in \Delta$ such that all fibers for $X_{(y, t)}$ for $t \in U$ are zero dimensional. Hence there are no $\pi_{1}$-small curves.

Case 2. Some $X_{(y, o)}$ are 1-dimensional. From the upper-semicontinuouty mentioned above and the fact that $g\left(X_{t}\right)$ can not be a point it follows that the set $S:=\left\{s \in g(X) \mid \operatorname{dim} g^{-1}(s)=1\right\}$ is a closed analytic subspace of $g(X)$. By shrinking $D$ we can assume that all irreducible components $S_{i}$ of $S$ pass through $g\left(X_{o}\right)$. This implies that all possible $\pi_{1}$-small curves on $X_{t}, t \in D$, lie in a family of curves in $X$ containing a curve in $X_{o}$. Fix a curve $X_{\left(y_{o}, o\right)}$ and an irreducible component $S_{i}$ of $S$. The family of curves $g: g^{-1}\left(S_{i}\right) \rightarrow S_{i}$, is a family whose central fiber contains no $\pi_{1}$-small curves. Theorem 3 then implies that there is an open neighborhood $y_{o} \in U \subset \boldsymbol{S} \boldsymbol{h}^{H}(X) \times D$, s.t. over $U \cap S_{i}$ does not have any not $\pi_{1}$-small curves. Since $S$ has only finitely many irreducible components, there is only a finite number of families to consider and so we can choose $U$ which does not contain any $\pi_{1}$-small curves. Now since $g\left(X_{o}\right)$ is compact the result follows.

Second Proof. We now give a second proof of Proposition 3.3 but under some additional hypothesis - we will assume that that $\pi_{1}\left(X_{o}\right)$ has finite torsion. In the second proof we use the volume tools (Theorem 2).

Claim 3.5 Let $X_{o}$ be a Kähler surface with $\rho: \pi_{1}\left(X_{o}\right) \rightarrow G L(n, \mathbb{C})$ an infinite linear representation. Assume that $X_{o}$ has a Stein universal cover and that $\pi_{1}\left(X_{o}\right)$ has finite torsion. Then any small Kähler deformation of $X_{o}$ has a large fundamental group.

Proof. We will use the relative $\rho$-Shafarevich morphism to control the volume of the $\pi_{1}$-small curves and then use Corollary 3.2 to get a contradiction.

Let $g=\boldsymbol{s} \boldsymbol{h}^{H} \times f: X \rightarrow \boldsymbol{S} \boldsymbol{h}^{H}(X) \times D$ be the same morphism as in the previous proof. Since $g$ is proper we can assume that by shrinking $\Delta$ the set of points $y \in g(X)$ with 1-dimensional $g^{-1}(y)$ is a finite union of irreducible closed sets, $\cup_{i} F_{i}$. Let $X_{F_{i}}=\operatorname{red}\left(g^{-1}\left(F_{i}\right)\right)$ be the reduced analytic space associated with $g^{-1}\left(F_{i}\right)$. The spaces $X_{F_{i}}$ are finite unions of irreducible components, hence to simplify the proof we can assume $X_{F_{i}}$ is irreducible. Let $\widehat{F}_{i}$ be the normalization of $F_{i}$ and $g_{\widehat{F}_{i}}: X_{\widehat{F}_{i}} \rightarrow \widehat{F}_{i}$ be the pullback to $\widehat{F}_{i}$ of $g: X_{F_{i}} \rightarrow F_{i}$.

According to [2, Theorem 3] the volume of the fibers of $g_{\widehat{F}_{i}}: X_{\widehat{F}_{i}} \rightarrow \widehat{F}_{i}$ relative to any hermitian metric over $X_{\bar{F}_{i}}$ is locally bounded over $\bar{F}_{i}$. We consider the metric induced from the Kähler metric on $X$. Due to the fact that there are only a finite number of $F_{i}$ implies that there is neighborhood $o \in U \subset D$ s.t all surfaces $X_{t}, t \in U$ have $\pi_{1}$-small curves with bounded volume. Recall that 
Corollary 3.2 says that the volume of the $\pi_{1}$-small curves is larger than any given number by making the deformation sufficiently small. Hence the result follows by contradiction.

Using the previous results we can now prove the stability of the Steiness of universal covers mentioned in the introduction:

Proof of Corollary 5. This result follows from looking at an intermediate cover of $X$, more precisely the infinite cover $X^{\rho}$ defined by $\operatorname{ker}(\rho)$. The starting point are the result of [12] stating that $X^{\rho}$ is holomorphic convex and the fact that this follows just from $\rho$ being an infinite linear representation. Hence $X_{t}^{\rho}$ being holomorphically convex is stable under deformations of $X$. To get the result one needs only to show that $X_{t}^{\rho}$ remains Stein for small Kählerian deformation of $X$, since unramified coverings (even infinite ones) of Stein varieties are still Stein.

By the previous paragraph, it only remains to show that having a $\rho$-large fundamental groups is stable for small Kählerian deformations of $X$. This however follows from Theorem 1

\subsection{Deformations of surfaces and $\pi_{1}$-small curves}

This subsection gives some results on $\pi_{1}$-small curves on surface deformations. We are going to use geometric bounds for the degree of curves on surfaces plus Theorem 2 to get information on the small curves that can appear along a deformation.

We also give a proof for the fact that a small deformation of a surface of general type whose universal covering is Stein has no rational curves $C$ with $C^{2} \leq 0$. We then note that this result plus a consequence of the Shafarevich Conjecture would rule out the appearance rational curves.

Proposition 3.6 Let $X_{o}$ be a surface of general type whose universal covering is Stein. Suppose $f: X \rightarrow D$ is a deformation of $X_{o}$ over the disc. Then for any $N \in \mathbb{N}$ there is a sufficiently small disc $o \in D(N) \subset D$ s.t. all $\pi_{1}$-small curves $C \subset X_{t}, t \in D(N)$, satisfy:

$$
\max \left\{g(C), C^{2}, \# \operatorname{im}\left[\pi_{1}(\widehat{C}) \rightarrow \pi_{1}\left(X_{t}\right)\right]\right\}>N,
$$

where $\widehat{C}$ is the normalization of $C$.

Proof. First note that the canonical class $K_{X_{o}}$ is ample since $\pi_{1}\left(X_{o}\right)$ being large rules out the existence of $(-2)$-rational curves. By shrinking the base $D$, if necessary, we can choose the relative canonical bundle $\omega_{X / D}$ as the relative ample line bundle required by Corollary 3.1. Thus we can find a disk $o \in D(N) \subset D$ s.t. all small curves $C \subset X_{t}, t \in D(N)$, satisfy:

$$
\max \left\{K_{X_{t}} \cdot C, \# \operatorname{im}\left[\pi_{1}(\widehat{C}) \rightarrow \pi_{1}\left(X_{t}\right)\right]\right\}>N
$$

Using the results of [11] in the spirit of the results of [3] we know that the degree of a curve $C$ having geometric genus $g(C)$ and selfintersection $C^{2}$ in a surface of general type $X$ is bounded by:

$$
K_{X} \cdot C<6 c_{2}(X)-2 c_{1}^{2}(X)+6 g(C)-6+C^{2}
$$


Since $6 c_{2}(X)-2 c_{1}^{2}(X)$ is a topological invariant of the surface it remains constant under deformations. Therefore the combination of (2) and (3) yields the desired conclusion.

The previous proposition implies that if any two of the three entries in (1) can be bounded the remaining entry must be very high.

The study of rational curves $C$ along small deformations is more attainable because in this case $\# \operatorname{im}\left[\pi_{1}(\widehat{C}) \rightarrow \pi_{1}\left(X_{t}\right)\right]=0$ (see the next remark) and so it is not necessary to give a bound for the order of the torsion subgroups of $\pi_{1}\left(X_{o}\right)$.

Remark 3.7 Let $C$ be a rational curve on a complex manifold $X$ and let $C^{\prime}$ be an irreducible component of the preimage of $C$ in the universal covering $\sigma: \widetilde{X} \rightarrow X$. Then $C^{\prime}$ is compact and the covering map $\sigma_{\mid C^{\prime}}: C^{\prime} \rightarrow C$ is of degree one. To see this we just notice that there is a map $\hat{\sigma}: \widehat{C}^{\prime} \rightarrow \widehat{C}$, between the normalizations of $C^{\prime}$ and $C$, that is by construction an unramified covering. Since $\widehat{C} \cong \mathbb{P}^{1}$ we have $\widehat{C}^{\prime}=\mathbb{P}^{1}$ and $\operatorname{deg}(\hat{\sigma})=1$.

Proposition 3.8 Let $X_{o}$ be a surface of general type whose universal covering is Stein. Then any small deformation of $X_{o}$ has no rational curves $C$ with $C^{2} \leq 0$.

Proof. It follows from the previous remark that $\# \operatorname{im}\left[\pi_{1}(\widehat{C}) \rightarrow \pi_{1}\left(X_{t}\right)\right]=0$. The hypothesis of the proposition bounds $C^{2}$, hence the conclusion is obtained using Proposition 3.6.

Remark 3.9 The Shafarevich Conjecture combined with Proposition 3.8 imply that if $X$ is a surface with large fundamental group then any small Kähler deformation of $X$ has no rational curves. If the universal covering $\widetilde{Y}$ of a surface $Y$ is holomorphically convex then there is Remmert reduction, $r: \widetilde{Y} \rightarrow S$. The compact curves $\widetilde{C}$ of $\widetilde{Y}$ are contained in the fibers of the reduction map $r$ and hence a $\pi_{1}$-small curve $C$ of $Y$ must have $C^{2} \leq 0$.

\section{References}

[1] A.Andreotti, E.Vesentini, On the pseudo-rigidity of Stein manifolds, Ann. Scuola Norm. Sup. Pisa (3) 16,1962,213-223.

[2] D.Barlet, Majoration du volume des fibres génériques et forme gomtrique du thorme d'aplatissement, Functions of several complex variables, IV (Sem. P. Lelong, 1978-1979), pp. 1-18, Lecture Notes in Math, 807, Springer, Berlin, 1980.

[3] F.Bogomolov, Families of curves on a surface of general type, Dokl. Akad. Nauk SSSR 236 (1977), no. 5, 1041-1044.

[4] F.Bogomolov, L.Katzarkov, Symplectic four manifolds and projective surfaces, Journal of topology and its applications, v. 88, n. 1-2, 1998, 79- 110.

[5] F.Campana, On twistor spaces of the class $\mathcal{C}$, J. Differential Geom. 33 (1991), no. 2, 541-549. 
[6] F.Campana, Remarques sur le revêtement universel des variétés kählériennes compactes, Bull. Soc. Math. France 122 (1994), no. 2, 255-284.

[7] K.Corlette, Nonabelian Hodge theory, Proc. Symp. Pure. Math. 54, part 2, 1993, 125-140.

[8] P.Eyssidieux, Sur la convexite holomorphe des reveteents lineaires reductifs d'une variete projective algebriques complexe, preprint, Univ. Paul Sabatier, Toulouse, 2000.

[9] M.Gromov, Kähler hyperbolicity and $L_{2}$-Hodge theory, J. Differential Geom. 33 (1991), no. $1,263-292$.

[10] M.Gromov, R.Schoen, Harmonic maps into singular spaces and p-adic superrigidity for lattices in groups of rank one, Publ. Math. I.H.E.S. 76 1992, 165-246.

[11] S.Lu, Y.Miyaoka Bounding curves in algebraic surfaces by genus and Chern numbers, Amer. J. Math. 119 (1997), no. 3, 487-502.

[12] L.Katzarkov, On the Shafarevich maps, Proc. of AMS, Vol. 62, Part 2, Alg. geometry, S. Cruz 1995, 173-217.

[13] L.Katzarkov, Nilpotent groups and universal coverings of smooth projective varieties, J. Differential Geom. 45 (1997), no. 2, 336-348.

[14] L.Katzarkov, T.Pantev, M.Ramachandran, Geometric factorization of linear fundamental groups, in preparation.

[15] L.Katzarkov, M.Ramachadran, On the universal coverings of algebraic surfaces, Ann. Sci. E.N.S., t. 31, f. 4, 1998, 525-536.

[16] J.Kollár, Shafarevich maps and plurigenera of algebraic varieties, Invent. Math. 113 1993, 165-215.

[17] J.Kollár, Shafarevich maps and automorphic forms, Princeton University Press, (1995).

[18] J.Kollár, Rational curves on algebraic varieties, Ergebnisse der Mathematik und ihrer Grenzgebiete, 3 Folge, 32. Springer-Verlag, Berlin, 1996.

[19] J.Kollár, Y.Miyaoka, S.Mori, Rationally connected varieties, J. Algebraic Geom. 1 (1992), no. 3, 429-448.

[20] N.Korevaar, R.Schoen, Global existence theorems for harmonic maps to non-locally compact spaces, Comm. Anal. Geom. 5 (1997), no. 2, 333-387.

[21] T.Napier, M.Ramachandran, Structure theorems for complete Kähler manifolds and applications to Lefschetz type theorems, Geom. Funct. Anal. 5 (1995), no. 5,809-851.

[22] T.Napier, M.Ramachandran, Fundamental groups, holomorphic convexity, and Barlet spaces, preprint, 2000.

[23] G.Pourcin, Théorème de Douady au-desus de S, Ann.Scuola.Nor.Sup.Pisa 23 (1969), 451459. 
[24] C.Simpson, Higgs bundles and local systems, Higgs bundles and local systems. Publ. IHES, Math. No. 75 (1992), 5-95.

[25] J.L. Verdier,Stratifications de Whitney et theoreme de Bertini-Sard, Invent. Math. 36 (1976), 295-312.

B. de Oliveira, University of Pennsylvania and Harvard University.

bdeolive@math.upenn.edu, bdeolive@math.harvard.edu

L. Katzarkov, UC Irvine.

lkatzark@math.uci.edu

M. Ramachandran, SUNY Buffalo

ramac-m@math.buffalo.edu 